\title{
Öklid 3-Uzayında Hasimoto Yüzeylerinin Paralel Yüzeyleri
}

\author{
Ali ÇAKMAK ${ }^{1^{*}}$ \\ ${ }^{1}$ Bitlis Eren Üniversitesi, Matematik Bölümü, Bitlis
}

\begin{abstract}
$\ddot{\mathbf{O z}}$
Bu çalışmada, ilk olarak Hasimoto yüzeyler ve paralel yüzeyler tanıtılmıştır. İkinci olarak, Hasimoto yüzeyler ve paralel yüzeylerle ilgili temel tanım ve teoremler verilmiştir. Bundan sonra, elde edilen paralel yüzeylerin birinci ve ikinci temel form katsayıları hesaplanmıştır. Böylece, Gauss ve ortalama eğrilikler bulunmuştur ve asıl yüzey ve paralelinin eğrilikleri arasındaki ilişkiler verilmiştir. Ayrıca, bu eğrilikleri kullanarak, bazı diferansiyel geometrik sonuçlar verilmiştir ve Hasimoto yüzeyi ve paralel yüzeyinin parametre eğrilerinin hangi şart altında geodezik, asimptotik veya eğrilik çizgisi olma durumları tartışılmıştır. Son olarak, eğer Hasimoto yüzeyini üreten eğri bir düzlem eğrisi ise o zaman paralel eğrinin eğriliği hesaplanmıştır. Bir örnek verilmiştir ve elde edilen eğriler Mathematica yardımıyla çizilmiştir.
\end{abstract}

Anahtar kelimeler: Hasimoto Yüzeyler, Paralel Yüzeyler, Gauss Eğriliği, Ortalama Eğrilik.

\section{Parallel Surfaces of Hasimoto Surfaces in Euclidean 3-Space}

\begin{abstract}
In this study, firstly Hasimoto surfaces and parallel surfaces are introduced. Secondly, basic definitions and theorems about Hasimoto surfaces and parallel surfaces are given. After that, the coefficients of the first and second fundamental forms of the obtained parallel surfaces are calculated. Thus, Gaussian and mean curvatures are found and the relations between the curvatures of the original surface and its parallel are given. In addition, using these curvatures, some differential geometric results are given and it is discussed that the parameter curves of Hasimoto surface and its parallel surface are geodesic, asymptotic or line of curvature under which condition. Finally, if the curve producing the Hashimoto surface is a plane curve, then curvature of the parallel curve is calculated. An example is given and the obtained curves are drawn with the help of Mathematica.
\end{abstract}

Keywords: Hasimoto Surfaces, Parallel Surfaces, Gaussian Curvature, Mean Curvature.

\section{Giriş}

3-boyutlu Öklid uzayda bazı yüzeyler integrallenebilir denklemler vasıtasıyla tanımlanabilir. Bu yüzey tiplerine örnek olarak verilebilecek yüzeylerden biri de Hasimoto yüzeyleridir. Bu yüzeyler, regüler bir $x$ uzay eğrisinin duman halka denklemi ya da lokalize indüksiyon denklemi (LIE) olarak bilinen

$x_{t}(s, t)=x_{s} \times x_{s s}=\kappa(s, t) b$

denklemine göre zamanla evirilerek elde edilir. Burada $x=x(s, t)$, eğri üzerindeki bir nokta için konum vektörü, $t$ zaman, $s$ eğrinin yay uzunluk parametresi, $\kappa$ eğrinin eğriliği, $b$ eğrinin binormali ve alt indisler verilen değişkenlere göre türevleri göstermektedir. 1972 de Hasimoto hareketli bir uzay eğrisi olarak ele alınan bu devinim denkleminin non-lineer Schrondinger (NLS) denklemine dönüştürülebileceğini gösterdi [1]. Bundan dolayı yüzey NLS ya da Hasimoto yüzeyi olarak tanımlanır. Uzay eğrilerinin devinimi, hiperyüzeylerin hareketi ve düzlem eğrilerinin devinimi birçok yazar tarafindan çalışılmıştır [2-5]. Vassilioulan ve Lisle, uzay eğrileri için varlık ve teklik teoremini

*Sorumlu yazar:alicakmak@yahoo.com

Geliş Tarihi: 30/04/2018 Kabul Tarihi: 01 /06/2018 
kullanarak, Serret-Frenet denklemlerinin integrasyonuyla Hasimoto yüzeylerini yapılandırmıştır [6]. Abdel-All ve Hussien, bu yöntemden farklı olarak, yüzeylerin temel teoremi ve Gauss-Weingarten denklemlerinin nümerik integrasyonuyla Hasimoto yüzeylerini temel form katsayıları ile yeniden yapılandırmıştır [7]. Erdoğdu ve Özdemir, Hasimoto yüzeylerini Minkowski 3-uzayda ele aldılar. Üç durumda bu yüzeylerin geometrik özelliklerini inceleyip, Gauss ve ortalama eğriliklerini buldular. Ayrıca, Minkowski 3-uzayda Hasimoto yüzeylerinin parametre eğrilerini karakterize ettiler [8].

Diğer yandan, bir yüzeyin yüzey normali boyunca sabit bir mesafede yeniden konumlandırılmasıyla elde edilen paralel yüzey kavramı da diferensiyel geometri de oldukça fazla ele alınan ve incelenen konulardan birisidir [9-13].

$\mathrm{Bu}$ çalışma da Hasimoto yüzeylerinin paralel yüzeyleri elde edilerek, asıl yüzey ile paraleli arasında ki çeşitli geometrik sonuçlar verilecektir.

\section{Materyal ve Metot}

Bu bölümde, Hasimoto yüzeyler ve paralel yüzeyler hakkında temel tanım ve teoremler verilecektir.

\subsection{Hasimoto Yüzeyler}

Daha önce duman halka denklemi olarak verilen

$x_{t}=x_{s} \times x_{s s}$

denkleminin bir çözümü $x=x(s, t)$ olarak ele alınabilir. Eğer $x(s, 0)$ yay uzunluğu ile parametrelendirilirse, her $t$ için $x=x(s, t)$ olur. Bu durumda eğer her $t$ için, binormal vektör alanı ile ele alınan ve (2) denklemini sağlayan hareketli uzay eğrisi $x=x(s, t)$, bir Hasimoto yüzeyi üretir [8]. Hasimoto yüzeyleri için sırasıyla $s$ ve $t$ parametrelerine göre Frenet çatı alanlarının elemanları arasındaki ilişki aşağıdaki denklemlerle ifade edilebilir:

$$
\begin{aligned}
& t_{s}=\kappa n, \\
& n_{s}=-\kappa t+\tau b, \\
& b_{s}=-\tau n
\end{aligned}
$$

ve

$t_{t}=-\kappa \tau n+\kappa_{s} b$,

$n_{t}=\kappa \tau t+\frac{\kappa_{s s}-\kappa \tau^{2}}{\kappa} b$,

$b_{t}=-\kappa_{s} t-\frac{\kappa_{s s}-\kappa \tau^{2}}{\kappa} n$

Burada, $s$ eğrinin yay uzunluk parametresi, $\{t, n, b\}$ Frenet çatısı ve $\kappa$ eğrinin eğriliği, $\tau$ ise eğrinin burulmasıdır. Eğrilik ve burulma fonksiyonları arasındaki ilişki ise

$$
\begin{aligned}
& \kappa_{t}=-2 \kappa_{s}-\kappa \tau_{s}, \\
& \tau_{t}=\kappa \kappa_{s}-2 \tau \tau_{s}+\left(\frac{\kappa_{s s}}{\kappa}\right)_{s}
\end{aligned}
$$

denklemleri ile verilir [7]. (5) denkleminin çözümü ile $c_{1}, c_{2}, c_{3}$ herhangi reel sabitler olmak üzere, 
$\kappa=2 c_{1} \operatorname{sech}\left(c_{2} t+c_{1} s+c_{3}\right)$,

$\tau=-\frac{c_{2}}{2 c_{1}}$

denklemleri olarak elde edilir [7].

$x=x(s, t)$ Hasimoto yüzeyi için $x_{s}=t$ ve $x_{t}=\kappa b$ yüzeye teğet vektörlerdir. Buradan hareketle birinci temel form katsayıları

$E=1, F=0, G=\kappa^{2}$

biçiminde hesaplanarak,

$I=d s^{2}+\kappa^{2} d t^{2}$

yazılabilir [7]. Yüzeyin birim normal vektör alanı; $n$ eğrinin birim normal vektörü olmak üzere,

$N=\frac{x_{s} \times x_{t}}{\left|x_{s} \times x_{t}\right|}=-n$

olur. Ayrıca, ikinci temel form katsayıları

$e=-\kappa, f=\kappa \tau, g=\kappa_{s s}-\kappa \tau^{2}$

olduğundan

$I I=-\kappa d s^{2}+2 \kappa \tau d s d t+\left(\kappa_{s s}-\kappa \tau^{2}\right) d t^{2}$

olur [7]. Böylece, Hasimoto yüzeyinin Gauss ve ortalama eğriliği sırasıyla,

$K=-\frac{\kappa_{s s}}{\kappa}$

$H=\frac{1}{2 \kappa^{2}}\left(\kappa_{s s}-\kappa^{3}-\kappa \tau^{2}\right)$

olarak elde edilir [7].

\subsection{Paralel Yüzeyler}

Tanım 2.1. $X_{1}$ ve $X_{2}$, 3-boyutlu Öklid uzayında iki yüzey ve $X_{1}$ in birim normal vektör alanı $n$ olsun. $r$ sabit bir sayı olmak üzere,

$f: X_{1} \rightarrow X_{2}, f(P)=P+r n_{P}$

olarak tanımlanan bir $f$ fonksiyonu varsa $X_{1}$ ve $X_{2}$ yüzeylerine paralel yüzeyler denir [9].

$X$ yüzeyi verildiğinde,

$X^{r}=\left\{P+r n_{P}: P \in M, r \in I R\right.$ ve $\left.r=s b t\right\}$ 
eşitliği ile verilen $X^{r}$ kümesi $X$ e paralel bir yüzeydir [14].

Teorem 2.1. $E^{3}$ de bir $X$ yüzeyinin paralel yüzeyi $X^{r}$ olsun. $P \in X$ noktasında $X$ nin Gauss ve ortalama eğrilikleri, sırasıyla, $K$ ve $H, f(P) \in X^{r}$ noktasında $X^{r}$ nin Gauss ve ortalama eğrilikleri de $K^{r}$ ve $H^{r}$ olsun. Bu durumda,

$K^{r}=\frac{K}{1-r H+r^{2} K}$

$H^{r}=\frac{H-2 r K}{1-r H+r^{2} K}$

$\operatorname{dir}[14-15]$.

Önerme 2.1. $\alpha: I \rightarrow E^{3}$ regüler bir düzlem eğrisi ve paraleli $\alpha^{r}$ olsun. $\alpha$ nın eğriliği $\kappa$ ve $1-r \kappa \neq 0$ olmak üzere, paralel eğrilerin eğrilikleri arasında;

$$
\kappa^{r}=\frac{\kappa}{|1-r \kappa|}
$$

bağıntısı vardır [15].

\section{Bulgular ve Tartışma}

Bu bölümde öncelikle Hasimoto yüzeyinin paralel yüzeyini elde edelim. Bunun için paralel yüzey tanımı gereği, $N$ yüzey normali olmak üzere,

$$
x^{r}(s, t)=x(s, t)+r N
$$

yazılabilir. Elde edilen bu paralel yüzeyin Gauss ve ortalama eğrilikleri arsında ki bağıntı için aşağıdaki teoremi yazabiliriz:

Teorem 3.1. $x=x(s, t)$ bir Hasimoto yüzey ve paraleli $x^{r}(s, t)$ olsun. Buna göre, paralel yüzeyin Gauss ve ortalama eğriliği sırasıyla $K^{r}$ ve $H^{r}$ ile gösterilmek üzere;

$$
K^{r}=-\frac{\kappa \kappa_{s s}}{\kappa^{2}-r \kappa_{s s}+r \kappa^{3}+r \kappa\left(\tau^{2}-r \kappa_{s s}\right)}
$$

ve

$$
H^{r}=\frac{\kappa_{s s}-\kappa\left(\kappa^{2}+\tau^{2}\right)+2 r \kappa \kappa_{s s}}{2\left(\kappa^{2}-r \kappa_{s s}+r \kappa^{3}+r \kappa\left(\tau^{2}-r \kappa_{s s}\right)\right)}
$$

olur. Burada, $\kappa$; Hasimoto yüzeyini üreten eğrinin eğriliğidir.

İspat: Paralel yüzeylerin Gauss ve ortalama eğrilikleri arasında ki ilişki Teorem 2.1 de verilmişti. Buna göre Hasimoto yüzeyinin (12) ve (13) denklemlerinde verilen Gauss ve ortalama eğrilikleri (15) ve (16) denklemlerinde yerlerine yazılırsa, kolaylıkla (19) ve (20) denklemleri elde edilir. 
Sonuç 3.1. Bilindiği gibi bir yüzeyin Gauss eğriliği sıfırsa yüzey açılabilirdir. O halde, Hasimoto yüzeyinin açılabilir olması için gerek ve yeter şart paralelinin açılabilir olmasıdır. Yani, $\kappa_{s s}=0$ olmalidir.

Sonuç 3.2. Bilindiği gibi bir yüzeyin ortalama eğriliği sıfırsa yüzey minimaldir. O halde, Hasimoto yüzeyinin minimal olması için gerek ve yeter şart $\kappa_{s s}=\kappa\left(\kappa^{2}+\tau^{2}\right)$ olmasıdır. Bu durumda paralel yüzeyin minimal olması için gerek ve yeter şart $\kappa_{s s}=\frac{\kappa\left(\kappa^{2}+\tau^{2}\right)}{1+2 r \kappa}$ olmasıdır.

Sonuç 3.3. Eğer bir yüzeyin Gauss ve ortalama eğrilikleri için $s$ ve $t$ parametrelerine göre kısmi türevler arasında $K_{s} H_{t}-K_{t} H_{s}=0$ eşitliği varsa yüzeye Weingarten yüzey denir. $O$ halde, Hasimoto yüzeyi için (12) ve (13) denklemlerinde kısmi türevler alınarak, bu sonuç kolaylıkla görülebilir. Benzer şekilde (19) ve (20) denklemlerinde $s$ ve $t$ parametrelerine göre kısmi türevler alınıp gerekli işlemler yapıldığında Hasimoto yüzeyinin paralelinin de Weingarten yüzey olduğu görülür.

Sonuç 3.4. Hasimoto yüzeyinin Gauss ve ortalama eğriliklerinin verildiği (12) ve (13) denklemleri oranlanırsa;

$$
\frac{\kappa}{H}=\frac{-\frac{\kappa_{s s}}{\kappa}}{\frac{1}{2 \kappa^{2}}\left(\kappa_{s s}-\kappa^{3}-\kappa \tau^{2}\right)}=-\frac{2 \kappa \kappa_{s s}}{\kappa_{s s}-\kappa\left(\kappa^{2}+\tau^{2}\right)}
$$

denklemi elde edilir. Eğer (21) denklemi sabit ise yüzey Lineer Weingarten yüzey olur. Benzer şekilde, (19) ve (20) denklemleri oranlanırsa;

$$
\frac{\kappa^{r}}{H^{r}}=-\frac{2 \kappa \kappa_{s s}}{\kappa_{s s}-\kappa\left(\kappa^{2}+\tau^{2}\right)+2 r \kappa \kappa_{s s}}
$$

elde edilir. Bu ifade de eğer sabit olursa, paralel yüzey de Lineer Weingarten yüzey olur. (21) ve (22) denklemlerinden denilebilir ki; Hasimoto yüzeyinin $K$ ve $H$ oranının sabit olması için gerek ve yeter şart $K^{r}$ ve $H^{r}$ oranının sabit olmasıdır.

Şimdi $x=x(s, t)$ yüzeyinin parametre eğrilerinin hangi durumlarda geodezik, asimptotik ve eğrilik çizgisi olduğunu gösterelim. Bunun için (3) denkleminden

$$
x_{s s}=t_{s}=\kappa n
$$

ve yüzey normalinin $N=-n$ olduğu göz önüne alınırsa, yüzeyin $s$-parametre eğrileri geodezik olur. Diğer taraftan, (1) denkleminde $t$ ye göre türev alınırsa,

$$
x_{t t}=(\kappa b)_{t}=\kappa_{t} b+\kappa\left(-\kappa_{s} t-\frac{\kappa_{s s}-\kappa \tau^{2}}{\kappa} n\right)
$$

elde edilir. Bu durumda $\kappa_{t}=0$ olması durumunda yüzeyin $t$-parametre eğrileri geodezik olur. Sonuç olarak, $t$-parametre eğrilerinin geodezik olması için $\kappa_{t}=0$ olması gerek ve yeterdir.

Teorem 3.2. $x^{r}=x^{r}(s, t)$ yüzeyi $x=x(s, t)$ ile verilen Hasimoto yüzeyinin paralel yüzeyi olmak üzere, $s$-parametre eğrilerinin geodezik olması için gerek ve yeter şart paralelinin de geodezik olmasıdır. 
İspat: $x=x(s, t)$ yüzeyinin paralel yüzeyi daha önce (18) denklemi ile belirtilmişti. $s$ ye göre kısmi türevler alınırsa,

$$
\begin{aligned}
& x_{s}^{r}=x_{s}+r N_{s}=(1+r \kappa) t-r \tau b \\
& x_{s s}^{r}=r \kappa_{s} t+\left(\kappa+r \kappa^{2}+r \tau^{2}\right) n-r \tau_{s} b
\end{aligned}
$$

elde edilir. Burada $x_{s s}^{r}$ in yüzey normaline paralel olması için $\kappa_{s}=\tau_{s}=0$ olmalıdır. Bu durumda $s-$ parametre eğrileri paralel yüzeyde geodezik olur.

$x=x(s, t)$ Hasimoto yüzeyinin $s$-parametre eğrilerinin asimptotik olması için (23) denklemi ve yüzey normalinin $N=-n$ olduğu göz önüne alınırsa, $\kappa=0$ seçilmesi durumunda yüzeyin $s-$ parametre eğrileri asimptotik olur. Diğer taraftan, (24) denkleminde, $n$ nin katsayısı sıfira eşitlenirse $t$-parametre eğrileri, $\kappa_{s s}-\kappa \tau^{2}=0$ için asimptotik olacaktır.

Sonuç 3.5. $x=x(s, t)$ Hasimoto yüzeyinin $s$-parametre eğrileri asimptotik ise $t$-parametre eğrileri de asimptotik olur.

Teorem 3.3. $x^{r}=x^{r}(s, t)$ yüzeyi $x=x(s, t)$ ile verilen Hasimoto yüzeyinin paralel yüzeyi olmak üzere, $s$-parametre eğrilerinin paralelinin asimptotik olması için gerek ve yeter şart ya $\kappa=\tau=0$ ya da $r=-\frac{\kappa}{\kappa^{2}+\tau^{2}}=$ sabit olmasidır.

İspat: Asıl yüzeyin asimptotik olma şartına benzer şekilde, $x^{r}=x^{r}(s, t)$ paralel yüzeyinin, $x_{s s}^{r}$ türev ifadesi olan (25) denkleminde normalin katsayısı sıfıra eşitlenirse $s$-parametre eğrilerinin asimptotik olma şart1

$\kappa+r \kappa^{2}+r \tau^{2}=0$

olarak elde edilir. Bu durumda $r$ sifirdan farklı bir sabit olduğundan ya $\kappa=\tau=0$ ya da $r=-\frac{\kappa}{\kappa^{2}+\tau^{2}}$ olur. Bu da ispatı tamamlar.

Bilindiği gibi bir yüzeyin parametre eğrileri, eğrilik çizgisi ise $F=f=0$ olur. O halde $x=x(s, t)$ Hasimoto yüzeyinin parametre eğrilerinin eğrilik çizgisi olması için (7) ve (10) denklemlerinden $F=f=\kappa \tau=0$ olması gerekir.

Şimdi de Hasimoto yüzeyini üreten eğrinin düzlem eğrisi olması durumunda paralelini veren teoremi ifade edelim:

Teorem 3.4. Hasimoto yüzeyini üreten eğrinin düzlem eğrisi olması durumunda eğrinin eğriliği $\kappa=2 c_{1} \operatorname{sech}\left(c_{2} t+c_{1} s+c_{3}\right)$ ise paralelinin eğriliği;

$\kappa^{r}=\frac{2 c_{1} \operatorname{sech}\left(c_{1} s+c_{2} t+c_{3}\right)}{\left|1-2 r c_{1} \operatorname{sech}\left(c_{1} s+c_{2} t+c_{3}\right)\right|}$

olur.

İspat: Hasimoto yüzeyini üreten eğrinin eğriliği (6) denklemlerinde verilmişti. Eğri düzlem eğrisi olduğundan $\tau=0$ olur. Ayrıca, Önerme 2.1 de (17) denklemi ile bir düzlem eğrisinin paraleli verilmişti. $\mathrm{Bu}$ durumda, (6) denklemi (17) denkleminde yerine yazılırsa kolaylıkla (26) denklemi elde edilir. 
Örnek 3.1. Hasimoto yüzeyini üreten eğrinin eğriliklerini $c_{1}=1, c_{2}=0, c_{3}=0$ için $\kappa=2 \operatorname{sech}(s), \tau=0$ olarak alırsak, paralelinin eğriliği $\kappa^{r}=\frac{2 \operatorname{sech}(s)}{|1-2 r \operatorname{sech}(s)|}$ olarak elde edilir. $r=1$ için bu eğrilere ait grafikler aşağıda gösterilmiştir.
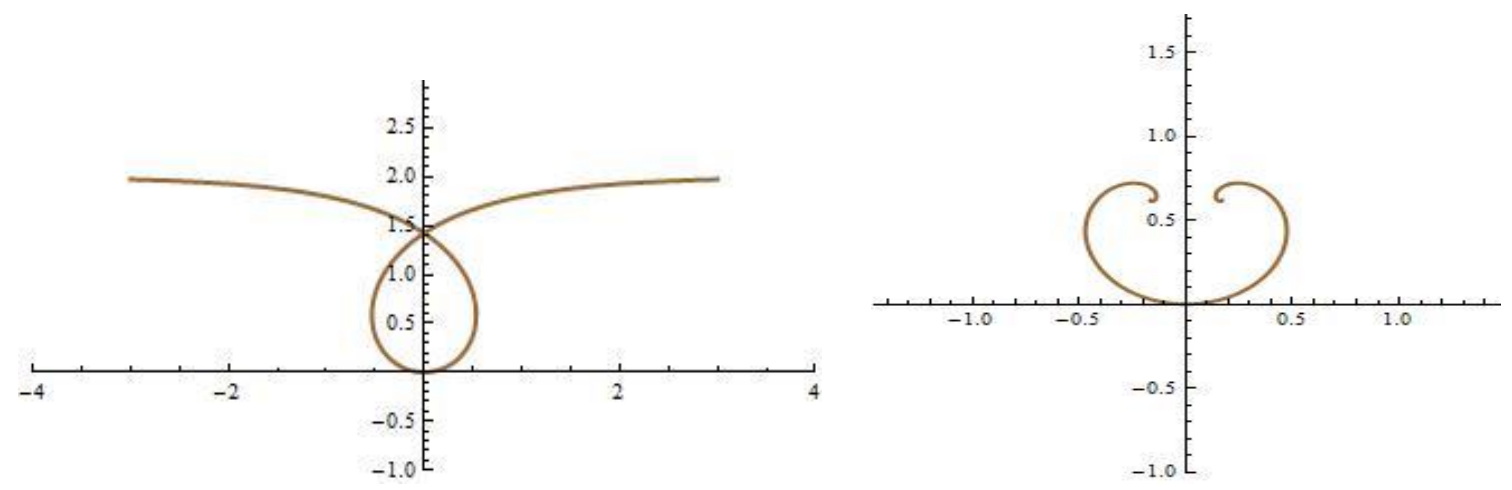

Şekil 1. $r=1$ için Hasimoto yüzeyini üreten eğri ve paraleli

\section{Sonuç ve Öneriler}

3-boyutlu Öklid uzayda Hasimoto yüzeyinin paraleli elde edilerek, paralel yüzeyin Gauss ve ortalama eğrilikleri, asıl yüzeyin eğrilikleri cinsinden (19) ve (20) denklemleri olarak hesaplanmıştır. Buradan, aşağıdaki sonuçlara ulaşılmıştır.

- Hasimoto yüzeyi açılabilir ise paraleli de açılabilirdir $\left(\kappa_{s s}=0\right)$.

- Hasimoto yüzeyi ve paralelinin minimal olma şartları sırasıla $\kappa_{s s}=\kappa\left(\kappa^{2}+\tau^{2}\right)$ ve $\kappa_{s s}=\frac{\kappa\left(\kappa^{2}+\tau^{2}\right)}{1+2 r \kappa}$ dir.

- Hasimoto yüzeyi ve paraleli Weingarten yüzeydir.

- Hasimoto yüzeyi için $\frac{K}{H}$ sabit ise paralelinde de $\frac{K^{r}}{H^{r}}$ sabit olup, Lineer Weingarten olurlar.

Daha sonra, Hasimoto yüzeyinin $s$ ve $t$-parametre eğrilerinin geodezik, asimptotik ve eğrilik çizgisi olma durumları incelenmiş ve aşağıdaki sonuçlar elde edilmiştir.

- Hasimoto yüzeyinde $s$-parametre eğrileri geodezik ise paralelinde de geodeziktir.

- Hasimoto yüzeyinde $s$-parametre eğrilerinin asimptotik olması için $\kappa=0$ olmalıdır. Diğer taraftan, paralelinde asimptotik olması için ya $\kappa=\tau=0$ ya da $r$ sıfirdan farklı bir sabit olmak üzere $r=-\frac{\kappa}{\kappa^{2}+\tau^{2}}$ olması gerekir.

Son olarak, Hasimoto yüzeyini üreten eğrinin düzlem eğrisi olması durumunda bu eğrinin paralelinin eğriliği (26) denklemi olarak elde edilmiştir.

Elde edilen bütün bulguların, 3-boyutlu Minkowski uzayda yeniden hesaplanması önerilebilir.

\section{Kaynaklar}

1. Hasimoto H. 1972. A Soliton on a vortex filament, J. Fluid Mech. 51: 477-485.

2. Abdel-All N. H, Abdel-Razek M. A, Abdel-Aziz H. S, Khali A. A. 2011. Geometry of evolving plane curves problem via lie group analysis, Studies in Mathematical Scinces, 2: 51-62. 
3. Abdel-All N. H, Hamad M. A. A, Abdel-Razek M. A, Khalil A. A. 2011. Computation of Some Geometric Properties for New Nonlinear PDE Models, Applied Mathematics, 2 (6): 666-675.

4. Abdel-All N H, Al-Dossary M. T. 2011. Motion of hyper surfaces, Assiut univ. Journal of Math. and Computer Science 40 (1): 91-104.

5. Abdel-All N. H, Mohamed S. G, Al-Dossary M. T. 2014. Evolution of generalized space curve as a function of its local geometry, Applied Mathematics, 5 (15): 2381-2392.

6. VassiliouIan P. J, Lisle G. 2000. Geometric approaches to differential equations. Cambridge University Press.

7. Abdel-All N. H, Hussien R. A, Youssef T. 2012. Hasimoto Surfaces. Life Science Journal, 9(3): 556-560.

8. Erdoğdu M, Özdemir M. 2014. Geometry of Hasimoto Surfaces in Minkowski 3-Space. Math. Phys. Anal. Geom, 17: 169-181.

9. Craig, T. 1883. Note on parallel surfaces, Journal f'ur die Reine und Angewandte Mathematik (Crelle's journal), 94: 162-170.

10. Patriciu, A. M. 2010. On some $\mathrm{H}_{3}$-helicoidal surfaces and their parallel surfaces at a certain distancein 3-dimensional Minkowski space. Annals of the University of Craiova, Maths. and Compt. Science Series, 37 (4): 93-98.

11. Ünlütürk Y, Özüsağlam E. 2013. On Parallel Surfaces in Minkowski 3-space. Twms J. App. Eng. Math. 3(2): 214-222.

12. Tarakçı Ö, Kızıltuğ S, Yaylı Y. 2013. On the curves lying on parallel surfaces in the Euclidean 3space $E^{3}$. Journal of Advanced Research in Dynamical and Control Systems, 5(3): 26-35.

13. Kızıltuğ S. 2017. Bertrand and Mannheim Partner-curves on Parallel Surfaces. Boletim da Sociedade Paranaense de Matemática, 35 (2): 159-169.

14. Hacısalihoğlu H. H. 1983. Diferensiyel Geometri, İnönü Üniversitesi Fen Edebiyat Fakültesi Yayınları, Ankara.

15. Gray A. 1998. Modern differential geometry of curves and surfaces with Mathematica, Crc Press, USA. 